\title{
Implementation of Mobile Point-of-sale Cashier Management System
}

\author{
Hsiu-Fen Tsai, ${ }^{1}$ Bao Rong Chang, ${ }^{2 *}$ Hsia-Chung Huang, ${ }^{2}$ and Chien-Feng Huang ${ }^{2 * *}$ \\ ${ }^{1}$ Department of Fragrance and Cosmetic Science, Kaohsiung Medical University, \\ 100, Shih-Chuan 1st Road, Kaohsiung 80708, Taiwan \\ ${ }^{2}$ Department of Computer Science and Information Engineering, National University of Kaohsiung, \\ 700, Kaohsiung University Rd., Nanzih District, Kaohsiung 811, Taiwan
}

(Received March 22, 2019; accepted July 22, 2019)

Keywords: online payment, third-party service platform, internet of things, mobile cash register

The traditional fixed point-of-sale cash register is not only expensive and bulky but also inconvenient to use. Therefore, the goal of this research is to realize a live-trading mobile cash register that can handle multiple online transactions concurrently and is capable of intelligent back-end data processing and analysis for transactions, especially applying Web technology and a back-end database for data exchange or third-party payment over the Internet. Regarding the Internet of Things, the wireless networking protocol Bluetooth is used to connect front-end devices to their smart-card-related accessories where near-field communication (NFC) technology is used to read data stored in a smart card for connection and data exchange between the smart card and the front-end devices. In this way, a point-of-sale system is not limited to being installed or fixed on a front desk; instead, it becomes a mobile cash register and accepts online payment, providing consumers with convenience and fast transactions.

\section{Introduction}

In this study, we aim to use a relatively low-cost approach to replace the expensive point-of-sale system in the market. Currently, the point-of-sale system in the market is a complete set, which also contains a format system. The system function also determines the sale price. Small stores or street vendors tend to carry inexpensive systems. Some well-design systems are expensive. The system developed in this study is cheaper than most products in the market and has more powerful functions and better support. The functions of the proposed system have been designed according to common operating modes, so that it can meet the needs of general merchants. Each transaction process leads to numerous business opportunities, including information that can improve performance; thus, the proposed system enables the analysis of big data to determine different models and the demand of consumers.

The device system proposed in this study includes three parts: (1) an Android device (2) PHP Web Service, and (3) a cash flow method. The integrated development environment (IDE) is Android Studio and the programming language is Java. A large amount of information

\footnotetext{
*Corresponding author: e-mail: brchang@nuk.edu.tw

** Corresponding author: e-mail: cfhuang15@nuk.edu.tw

https://doi.org/10.18494/SAM.2019.2427
}

ISSN 0914-4935 (C) MYU K.K. https://myukk.org/ 
accessible on the Internet can be acquired, and updated technologies can be easily found on the Internet. The back-end server uses a virtual host set up by Proxmox Virtual Environment $(\mathrm{PVE})^{(1)}$ to execute WebService, written using PHP as the platform for the data exchange between the front and back-end platforms. ${ }^{(2)}$ Finally, in terms of the cash flow method, money transactions can only be processed with data exchange specifications published by various financial institutions. As the transactions involve the confidential security of financial institutions, the internal operation mode is not public. Only a particular information exchange interface is accessible to the public. Developers can simply communicate with it for cash flow. However, if the financial institution fails to provide an Android communication interface, the on-line transaction definitely does not work here. If the communication interface of another programming language is provided, there are other ways to solve this problem. The research process is shown in Fig. 1.

\section{Related Work}

The near-field communication (NFC) technology ${ }^{(3,4)}$ in mobile phones can read and write on a Mifare Card ${ }^{(5,6)}$ made using RFID technology. The i-Cash EasyCard has been launched in cooperation with President Chain Store in 2009 having such specifications. ${ }^{(7,8)}$ If this card can be combined with a merchant's mobile cashier system, the operating efficiency can be improved considerably and consumers become interested in using this card when making purchases, together with the Mifare membership card developed by merchants themselves, which records

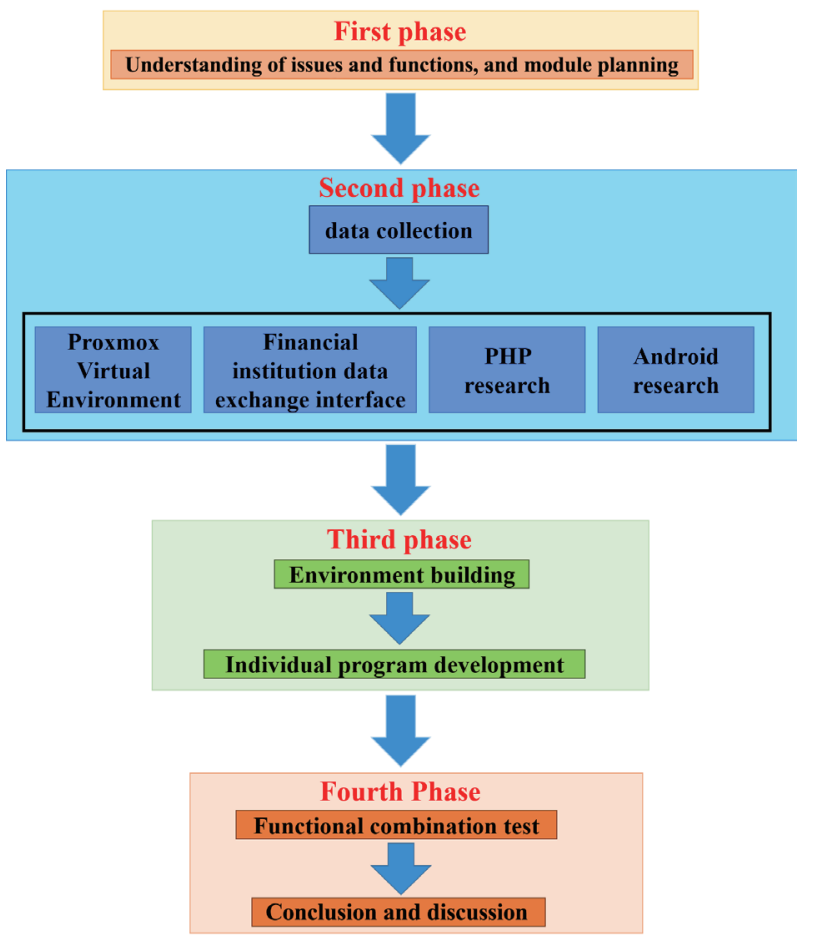

Fig. 1. (Color online) Research process. 
the membership points gained from their purchases. This system allows consumers themselves to control the information and use of points. A large data database can also be produced through the combination of basic data and the purchase records of members so as to simulate the preferences and consumption habits of consumers, launch discount activities for members, greatly stimulate consumer demand, and increase the operating performance. The data storage block of a Mifare card is shown in Fig. 2. Statistical data have shown that the number of mobile phones with NFC functions would increase by $30 \%$ after 2015 . Hence, NFC technology is essential in smart phones. ${ }^{(9)}$ The growth trend is shown in Fig. 3. ${ }^{(10)}$

Bluetooth communication technology ${ }^{(11)}$ is used in this study to connect to a thermal printer. Bluetooth communication is a type of short-range wireless communication. ${ }^{(12)}$ The Bluetooth transmission protocol is shown in Fig. $4 .{ }^{(13)}$ As Bluetooth uses the peer-to-peer online mode, its setting is simple and its data transmission is fast. The online distance is at least $10 \mathrm{~m}$. This technology is therefore very convenient to use, and its transmission of text and data is rapid and robust. ${ }^{(14)}$ It is thus a good choice for transmitting and printing documents and other information. Figure 5 shows the advantages and disadvantages of Bluetooth and NFC in terms

\begin{tabular}{|c|c|c|c|c|c|}
\hline \multicolumn{5}{|c|}{ Diagram of Data Strong Block } \\
\hline & Block 0 & Block 1 & Block 2 & Block 3 \\
\hline Sector 0 & UID & data & data & Key - Access bit \\
\hline Sector 1 & data & data & data & Key - Access bit \\
\hline Sector 2 & data & data & data & Key 、 Access bit \\
\hline : & $:$ & $:$ & $:$ & $:$ \\
\hline Sector 14 & data & data & data & Key - Access bit \\
\hline Sector 15 & data & data & data & Key - Access bit \\
\hline & & & \multicolumn{3}{c}{ UID: unique identifier } \\
\hline
\end{tabular}

Fig. 2. (Color online) Diagram of data storage block.

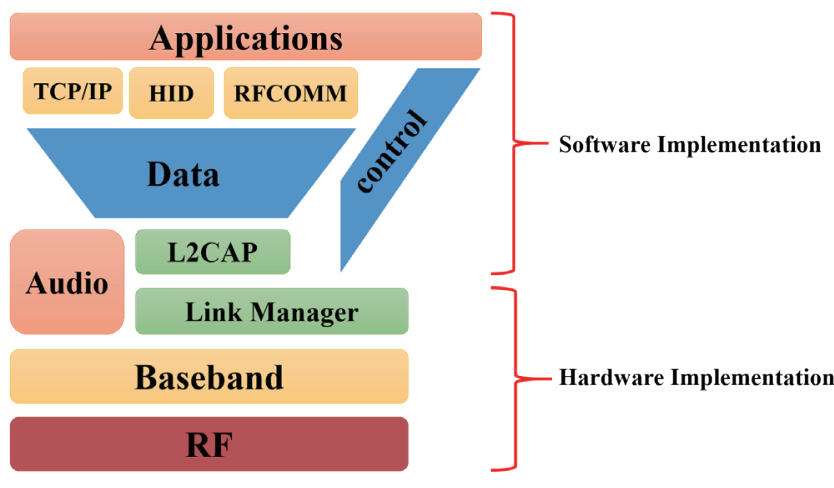

Fig. 4. (Color online) Schematic diagram of Bluetooth transmission protocol.

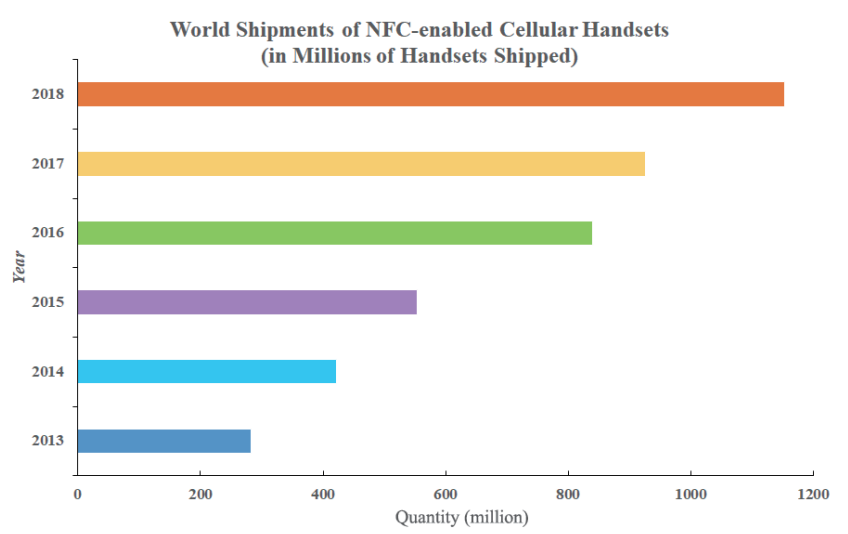

Fig. 3. (Color online) Predicted growth of mobile phones.

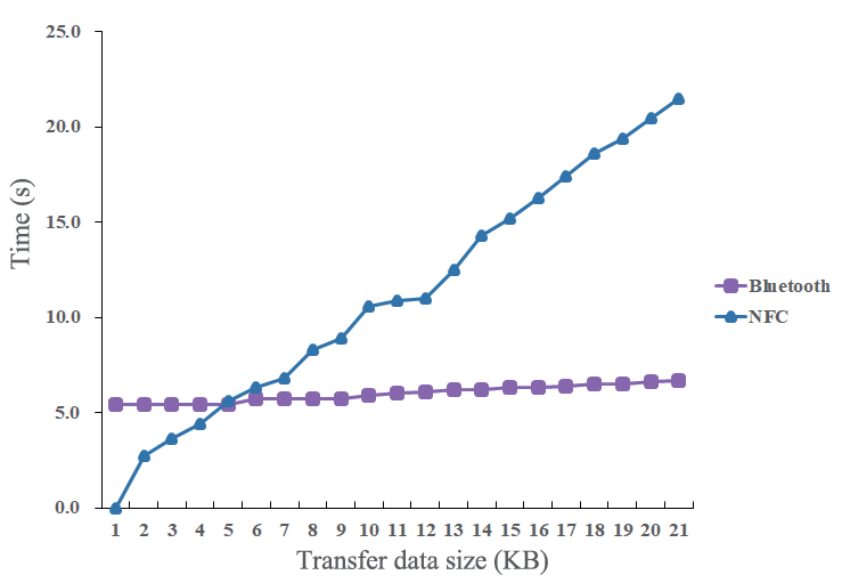

Fig. 5. (Color online) Actual transmission times of NFC P2P and Bluetooth. 
of actual transmission time. ${ }^{(15)}$ When the transmission capacity is less than $4 \mathrm{kB}$, the NFC speed is higher than the Bluetooth speed; however, when the transmission capacity is greater than $4 \mathrm{kB}$, the Bluetooth speed is much higher than the NFC speed. Although the capacity of a printed text is small, the text transmission speed decreases significantly if the amount of transaction data increases.

For barcode identification, ${ }^{(16)}$ barcodes and QR codes have been widely used in the market, and the use of barcodes and QR codes for identification has become popular. A CCD sensor is also used to receive reflected image as the basic image for identification. The clarity and resolution of the image directly affect the accuracy of identifying results. Three simple steps for barcode identification are to acquire the original image, remove the noise, and capture the image block having the barcode. After image binarization and clarity processing, the granularity of a barcode, or a QR code, can be analyzed and then quantified as the corresponding decimal value. All up-to-date smart phones possess the required devices and high-resolution cameras; thus, they can be used for barcode and QR code identification. ${ }^{(17)}$ As there are free and effective identification modules on the Internet, the operation process is not described in detail here.

The http data exchange framework is widely used in web-based services or mobile applications, ${ }^{(18)}$ and the back-end server host uses the Apache architecture. The user end needs packages provided by Apache to obtain the contents on the Internet by the GET or POST method. In this study, we use such an architecture to change the contents of the webpage into the $\mathrm{xml}$ data format and then analyze its Tag to obtain different types of information.

Regardless of the type of system, a small database in a cloud system is required to record the related data for different uses. In an Android system, an in-cloud SQLite database ${ }^{(19)}$ is used to store the user's chat records in the design and implementation of online instant messaging software on the Android platform. The applied Android content provider monitors the changes in the database and displays chat records on the screen of mobile phones in real time to realize one-on-one real-time information exchange. ${ }^{(20)}$ The daily consumption records can be stored in the proposed system to obtain the real-time statistics of sales amount or performance within a certain period of time offline. Moreover, the iPOS system we proposed here can also be used to record login accounts set by the background.

\section{System Implementation}

\subsection{Architecture diagram for main system}

First, start the point-of-sale system app installed in a mobile phone; then, the system begins to run after logging into the system. The point-of-sale system has the following three main functions: (1) It runs statistics and records trading items and sales amount. (2) It allows data exchange through the http communication protocol, including the uploading of the communication mode of transaction data and cash flow. (3) It controls the peripheral hardware functions of a smart phone, such as the Bluetooth printing, reading, and writing of a Mifare induction card, and the barcode scanning of a camera. The system architecture is shown in Fig. 6. 


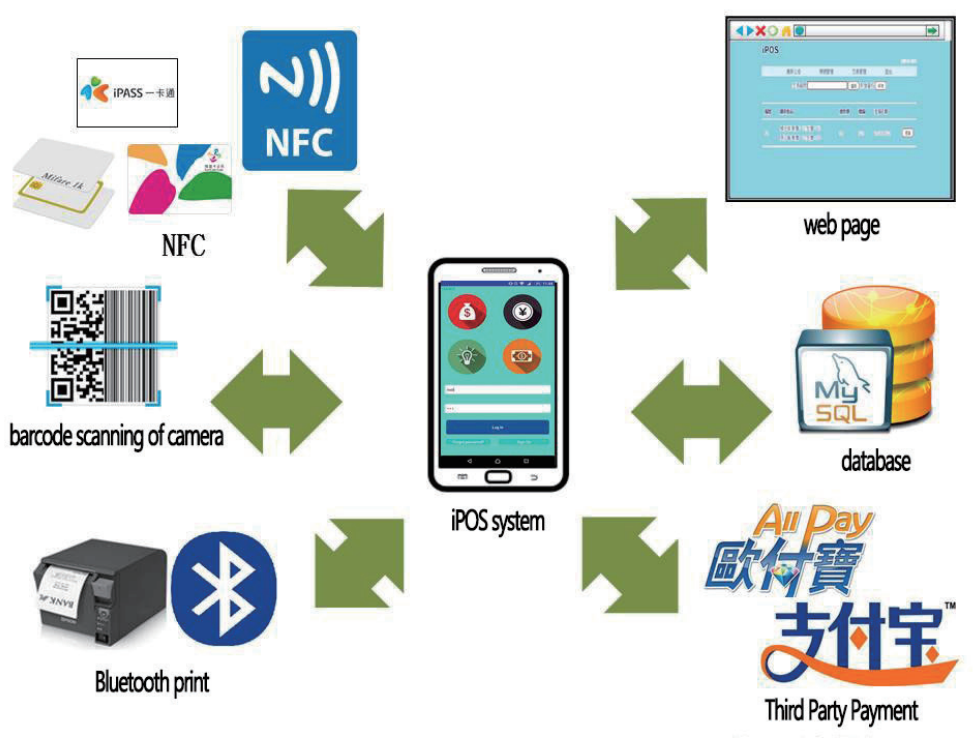

Fig. 6. (Color online) System architecture diagram.

\subsection{Introduction to development tool}

In this study, we adopt Android Studio 2.0 as the tool for the development of the system. Such a tool was newly launched by Google, the formal version 1.0 was introduced in December 2014 for free download and use, and the following is the simple procedure for setting up an environment installation. ${ }^{(21,22)}$

(1) Install JDK as the main language of Android using Java (Oracle Java JDK). The download location is http://www.oracle.com/technetwork/java/javase/downloads/index.html.

(2) Download and install Android Studio Tools.

(3) A home screen will appear to make users choose to start a new project or import an old project after the first execution. A screenshot is shown in Fig. 7.

(4) Open Android Studio to download Android SDK Tools and other relevant information.

(5) Please refer to the installation steps in detail. ${ }^{(21)}$

\subsection{Transaction process over iPOS}

Input the amount first, enter the product number, press the enter key, press the checkout button to choose the payment method, and then press the print button after the end of input. Among these steps, the input of the product number can both adopt the manual input or barcode scanning. The transaction process is shown in Fig. 8. A picture of each function is shown in Fig. 9, that is, the manual input of the amount. The manual input of the product number or the scanning of the barcode is shown in Fig. 10. The product deletion function input is shown in Fig. 11. Press the product button for a long time to delete. The checkout function is shown in Fig. 12. Press the checkout button to choose the payment method and then the checkout 


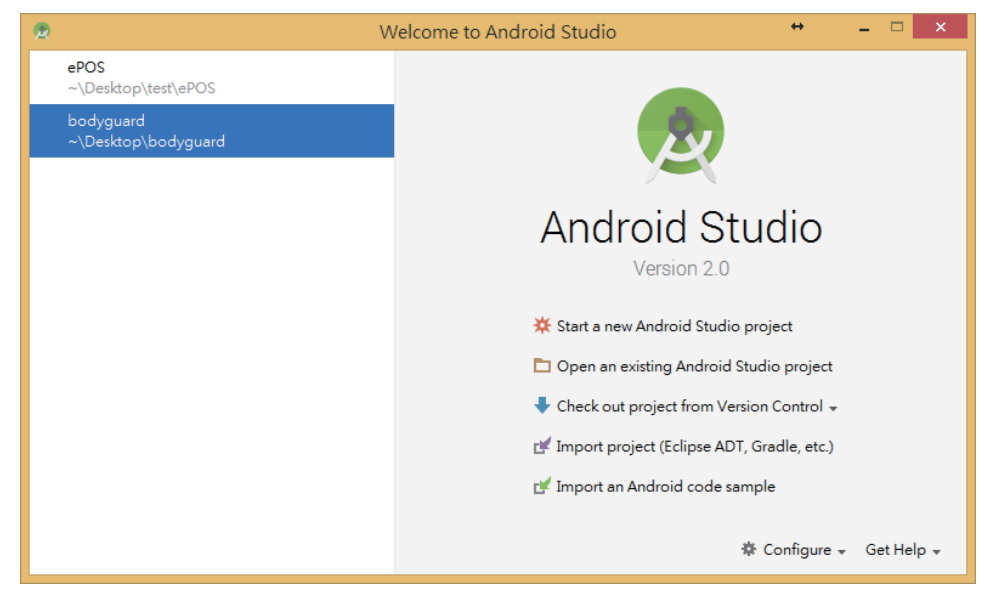

Fig. 7. (Color online) Home screen of Android Studio.

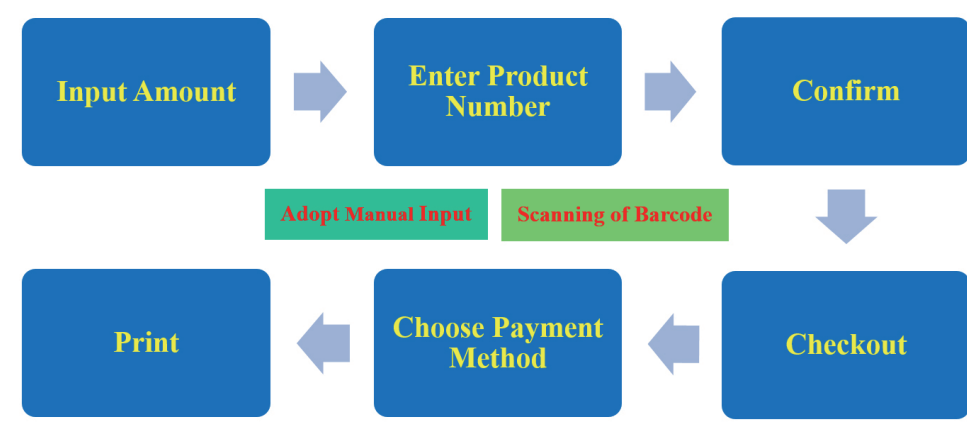

Fig. 8. (Color online) Transaction process diagram.

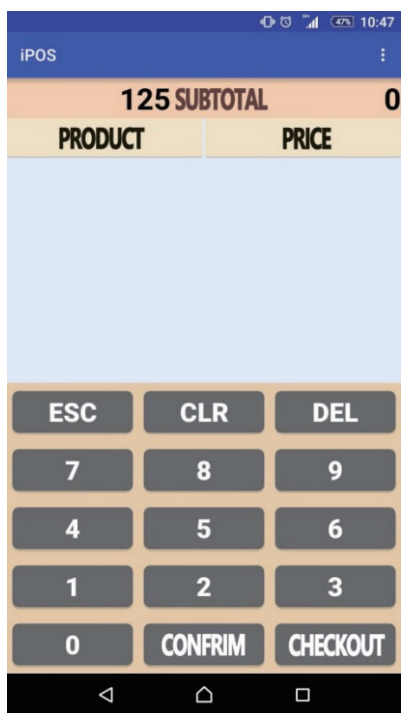

Fig. 9. (Color online) Manual input of amount.

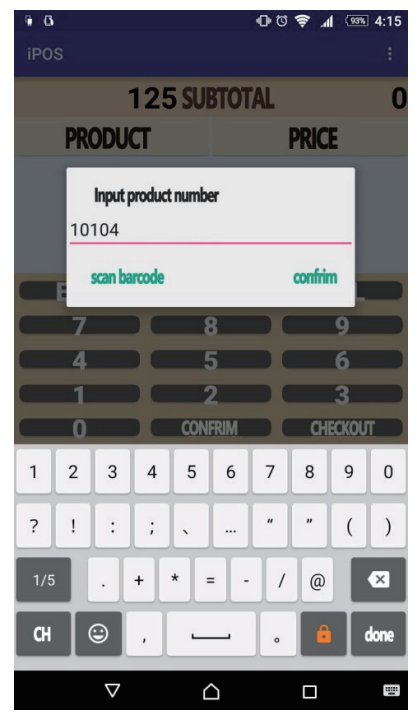

(a)

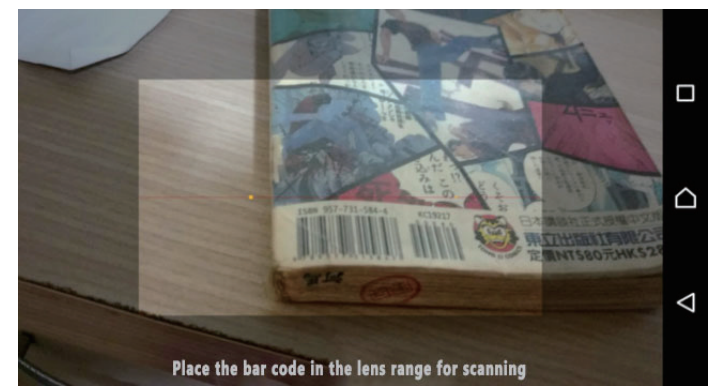

(b)

Fig. 10. (Color online) Manual input of product number or scanning of barcode. 


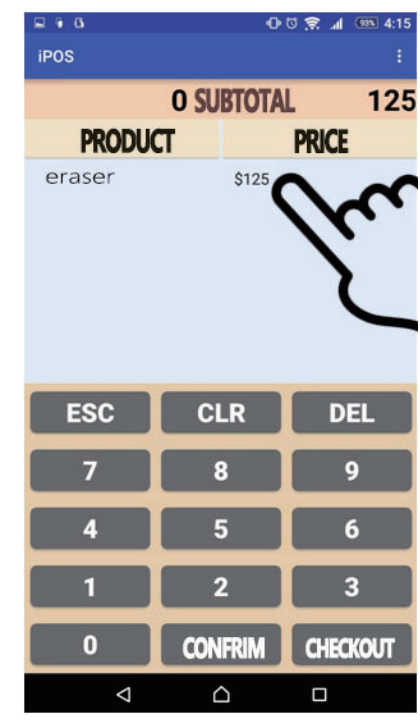

(a)

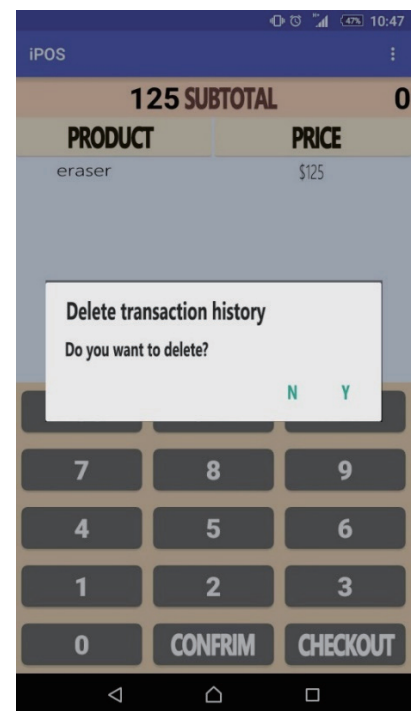

(b)

Fig. 11. (Color online) Product deletion function.

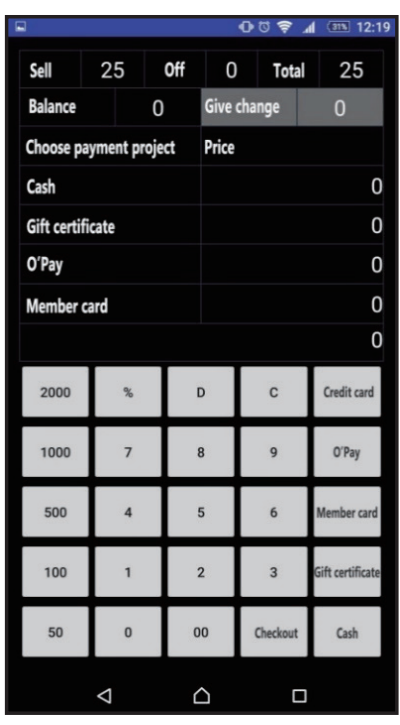

(a)

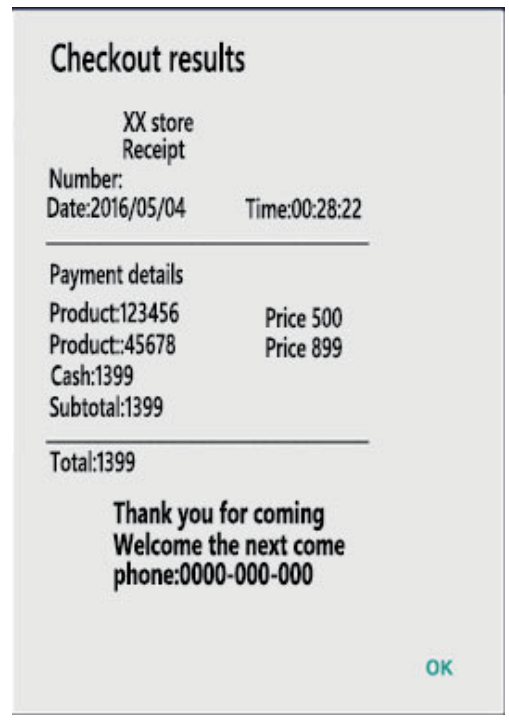

(b)

Fig. 12. (Color online) Checkout function.

number to print the receipt. The multiple functional menu selection is shown in Fig. 13. There are selection items to set the sales system or the functions of newly added products and sales return. The sales return function is shown in Fig. 14. Sales return is divided into whole return or partial return. Whole return means bringing the purchased products, receipt and electronic invoice to input the transaction number, delete the sales records, and return the payment to the consumers. In the case of partial return, we need to return all of the payment first and then 


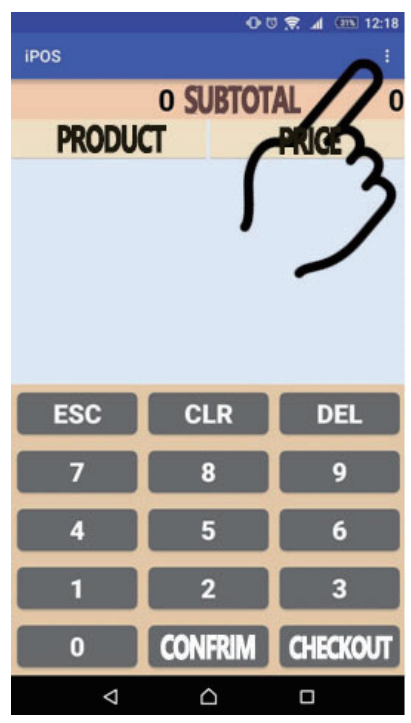

(a)

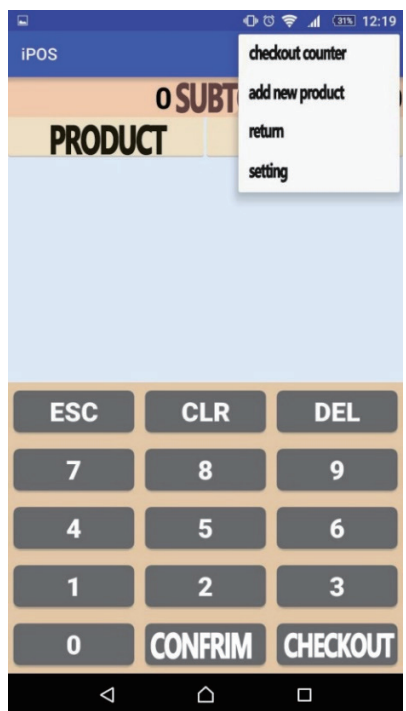

(b)

Fig. 13. (Color online) Multiple functional menu selection.

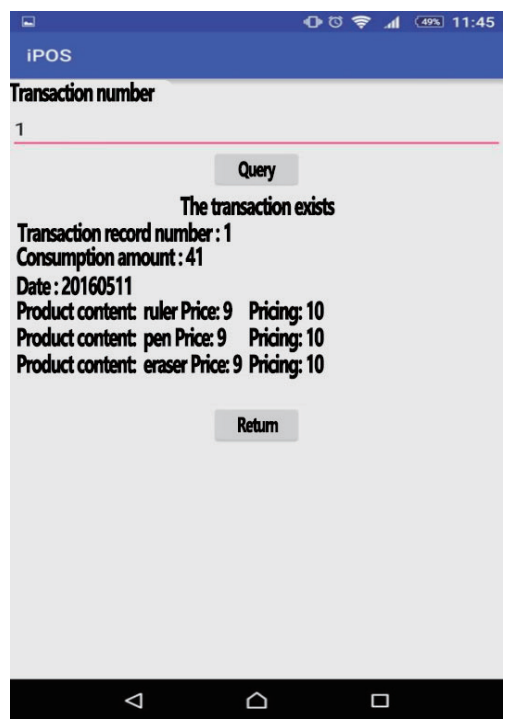

(a)

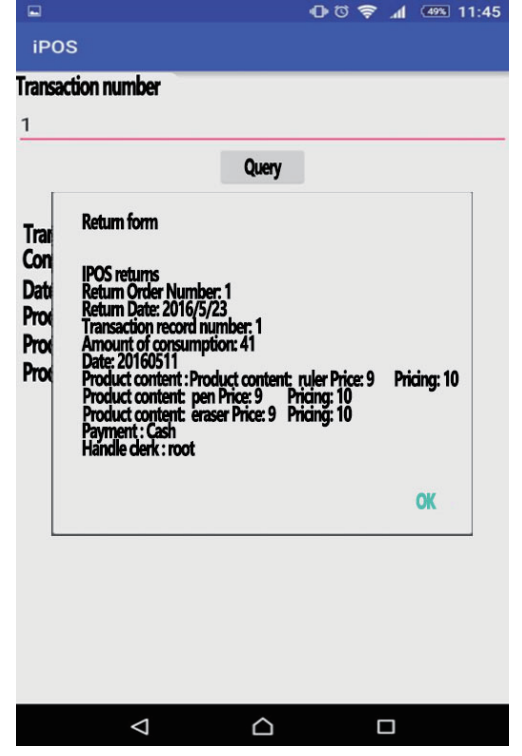

(b)

Fig. 14. (Color online) Sales return function.

input the price of products desired into a new sales record. Then, the amount returned to the consumer is re-calculated. The backstage management system is shown in Fig. 15. In the Login Page, input the account number and password to enter the backstage management system. The account management is shown in Fig. 16. Addition, deletion, and modification are allowed in the account management of the sales system here, and the staff can use the accounts listed here to log into the iPOS system. The transaction history record and management page are shown in 


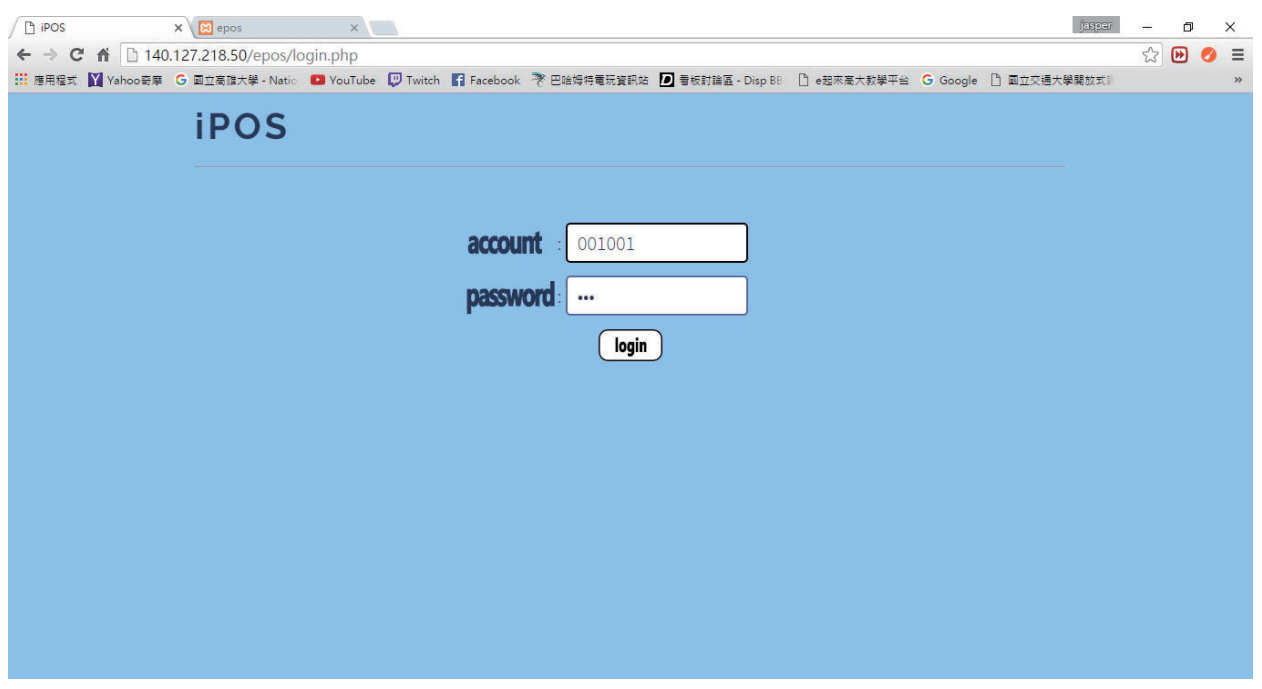

Fig. 15. (Color online) Login page.

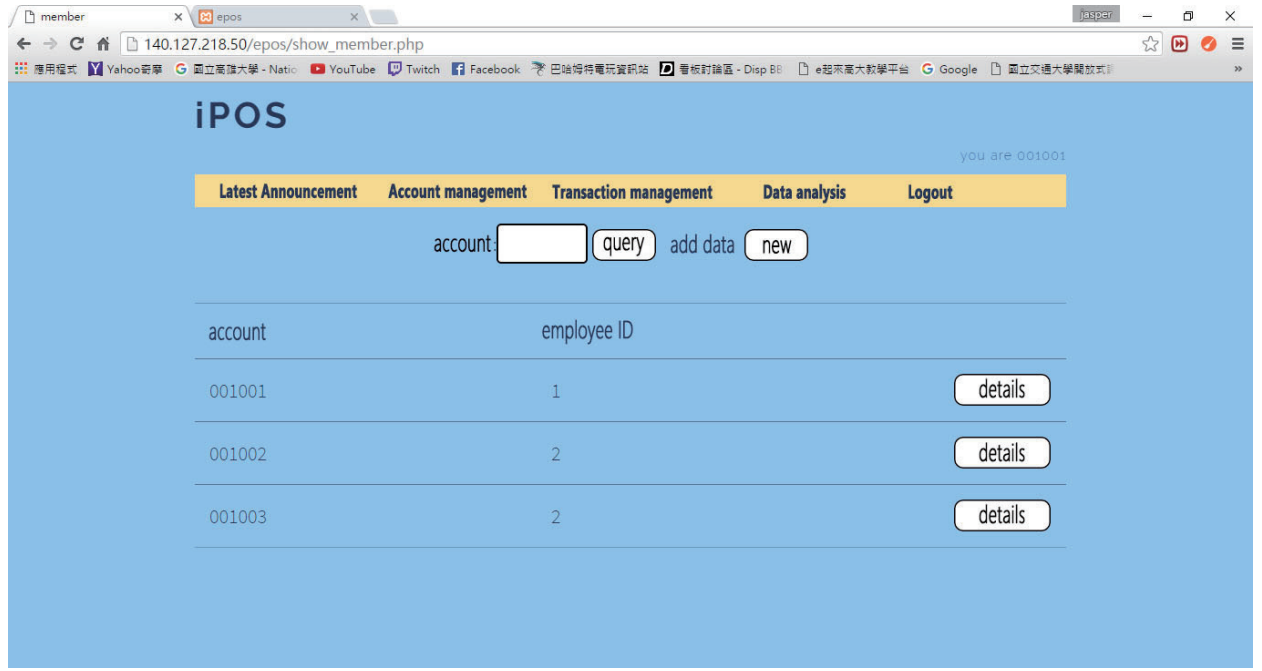

Fig. 16. (Color online) Account management page.

Fig. 17. We can see every transaction history record and amount here and the detailed product projects purchased after clicking on the "Detailed Record". A detailed product project is shown in Fig. 18. Click on the "Detailed Record" to see a purchased detailed product project.

\subsection{Transaction process of electronic purse}

The electronic purse is issued by merchants themselves: with this purse, consumers can store money first and then write the amount into the card and background database, and consumers can enjoy discounts in the case of purchases using this card. The process of payment with the electronic purse is shown in Fig. 19. 


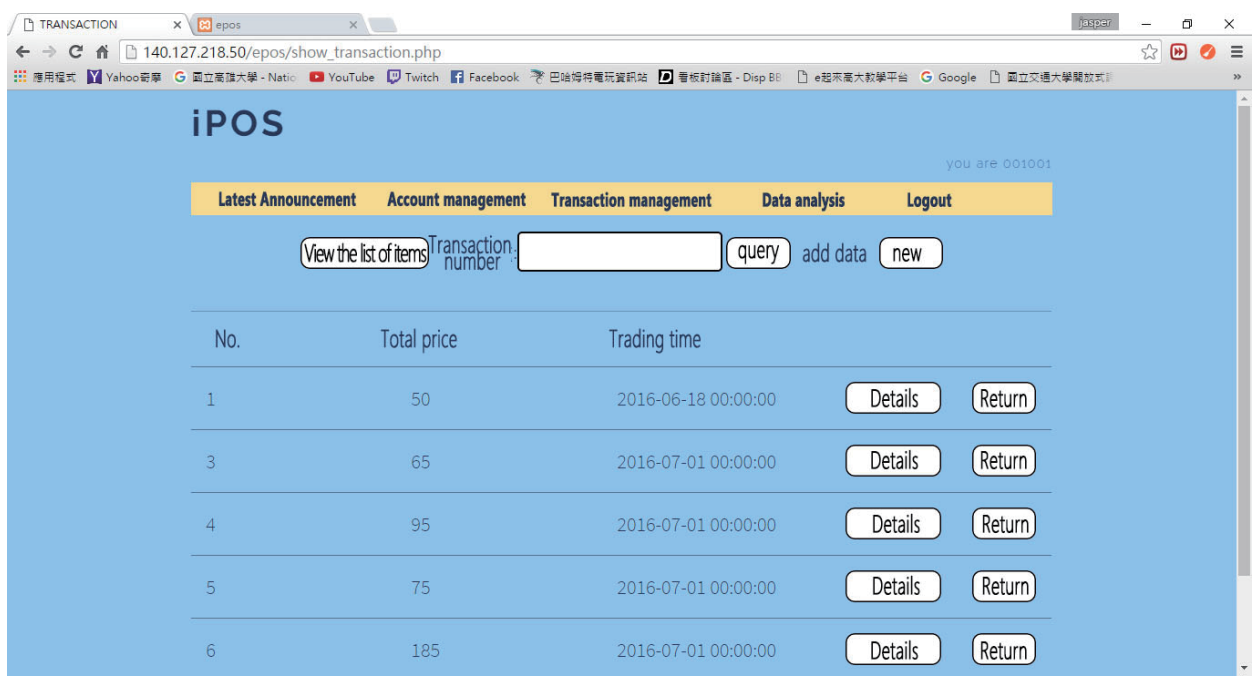

Fig. 17. (Color online) Transaction history record and management page.

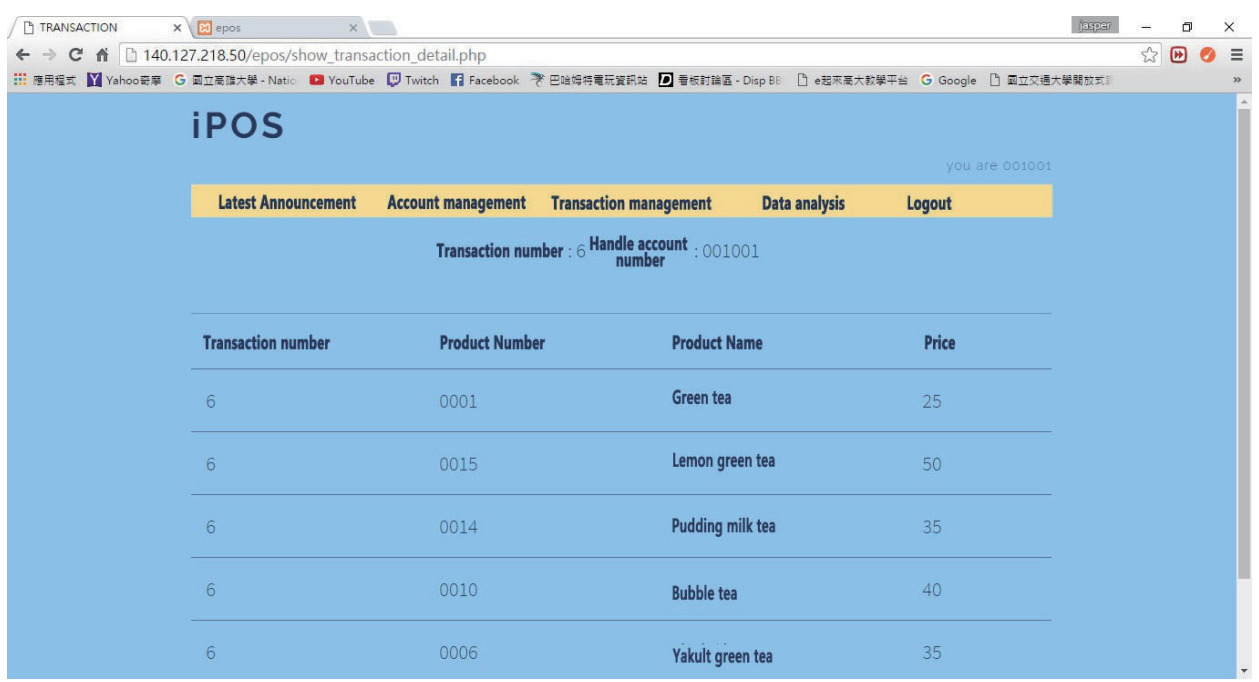

Fig. 18. (Color online) Detailed product project page.

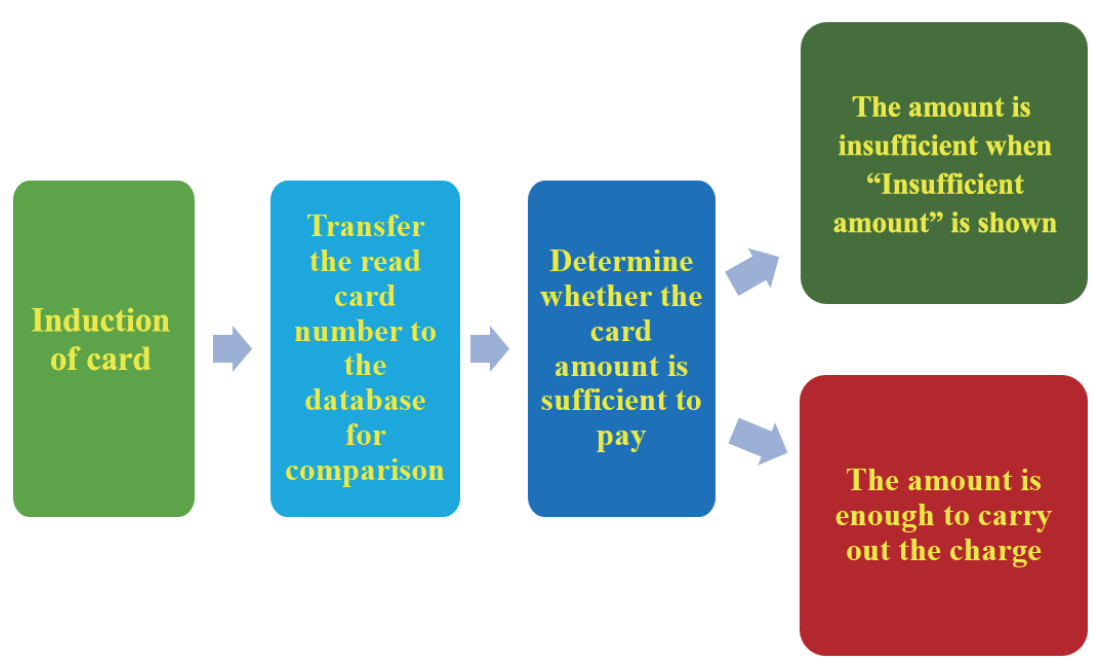

Fig. 19. (Color online) Payment process diagram for electronic purse. 


\section{Experimental Results and Discussion}

\subsection{Implementation of payment system of O'Pay}

O'Pay, ${ }^{(23)}$ a mobile payment, provides the data transmission interface (PHP API) to generate a two-dimensional barcode on the web page of a mobile sales system. It asks consumers to scan the code and leads them to the payment page of O'Pay where customers can choose the payment mode they want, such as credit card, online ATM, or O'Pay account payment. The cash flow process of a credit card is shown in Fig. 20.

\subsection{Real transaction over iPOS}

The picture of a two-dimensional barcode generated by the sales system is shown in Fig. 21. The picture of the consumer's payment portal for O'Pay is shown in Fig. 22. In credit card payment, relevant information and complete identification verification are input to finish the payment as shown in Fig. 23. The picture of completing the transaction is shown in Fig. 24,

Cash Flow Process of Credit Card

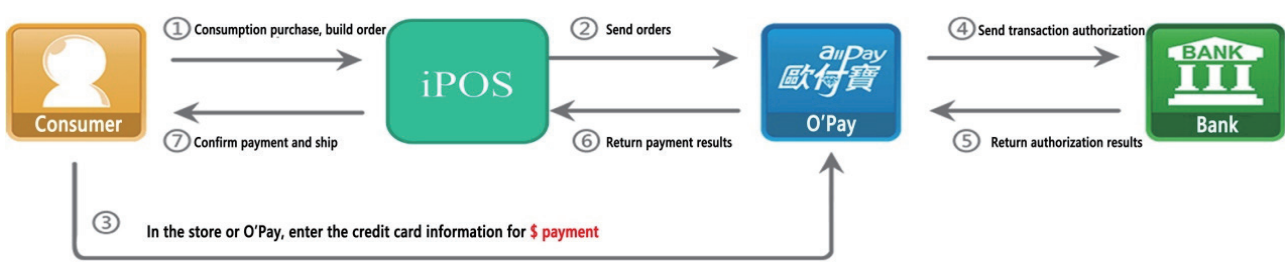

Fig. 20. (Color online) Cash flow process of credit card.

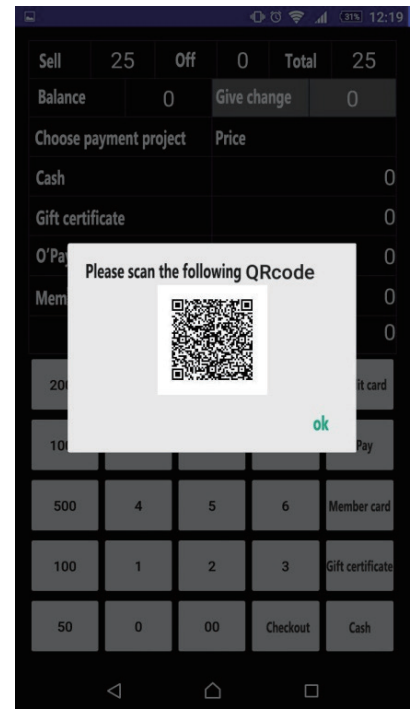

Fig. 21. (Color online) Two-dimensional barcode generated by sales system. 


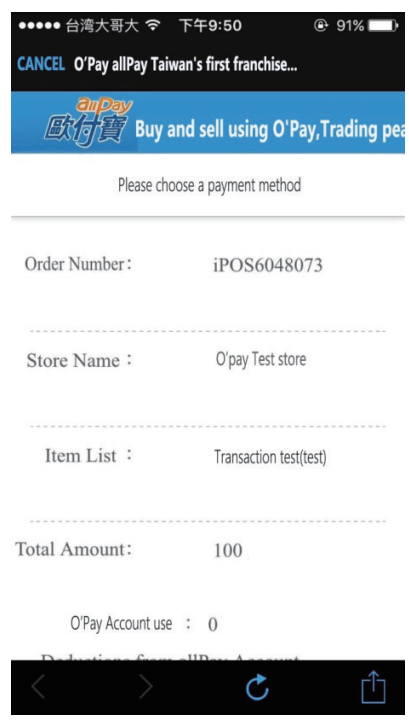

(a)

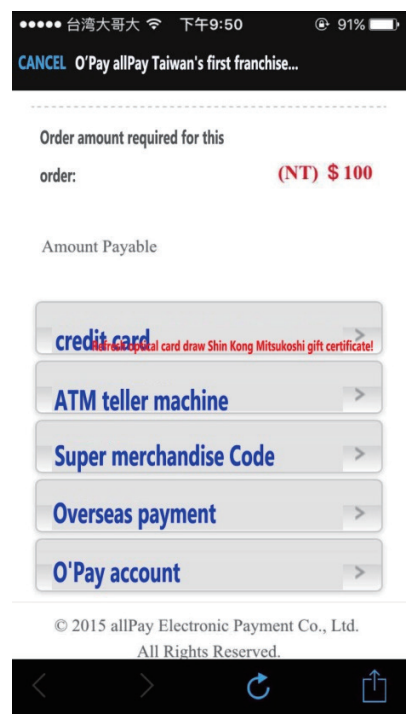

(b)

Fig. 22. (Color online) Picture of consumer's payment portal for O'Pay.

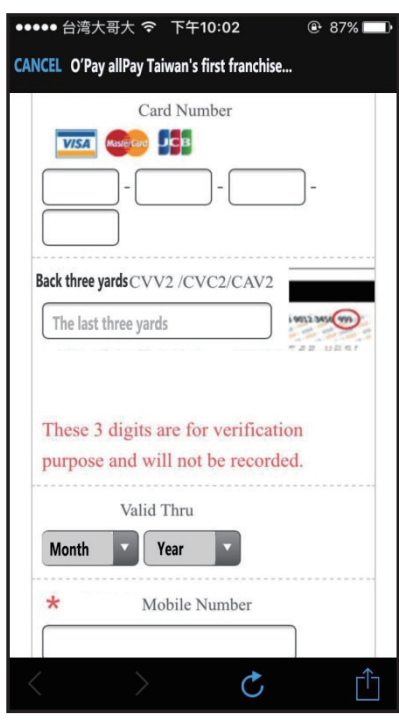

(a)

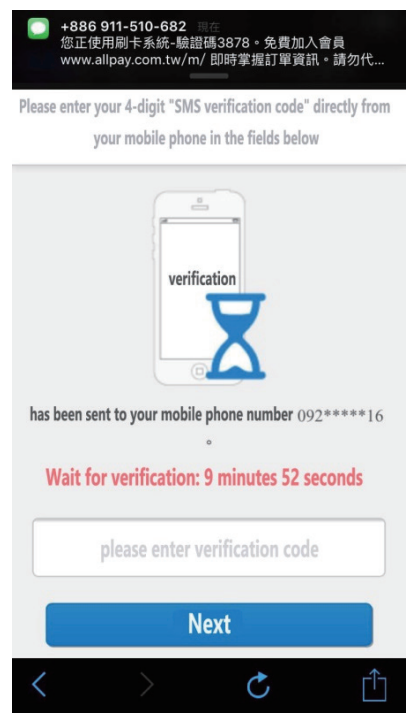

(b)

Fig. 23. (Color online) Picture of credit card input and page of identification verification.

where the sales system can determine that the payment for this order has been completed, checkout can be done, and the product can be given to consumers.

In fact, the mobile cash register cannot print an electronic invoice at present, because it must get the permission from the Ministry of Finance in Taiwan. ${ }^{(24)}$ It is feasible to print an electronic invoice by opening a personal store and its annual turnover reaches a certain threshold. The hardware peripheral that can support mobile phones is currently not comprehensive. Although there are magnetic stripe card readers for mobile phones, their security cannot be identified by 

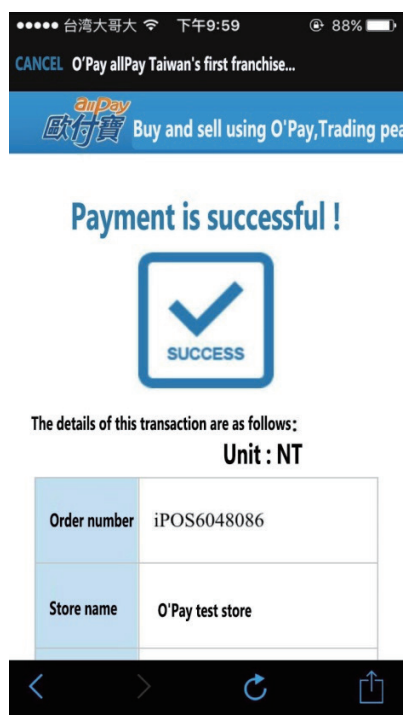

(a)

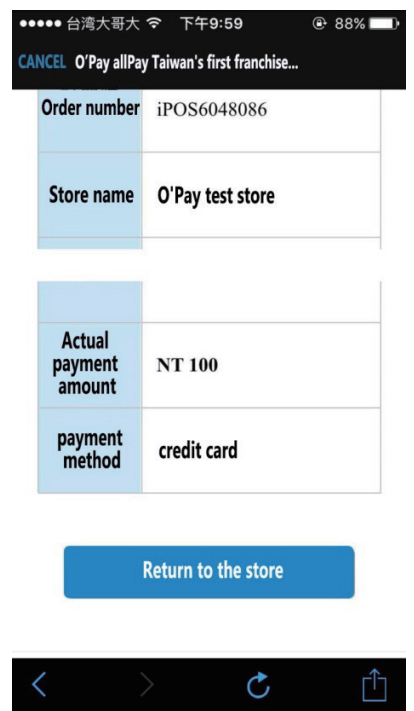

(b)

Fig. 24. (Color online) Picture of completing transaction.

banking institutions. ${ }^{(25)}$ If you really want to achieve cash flow directly with banks, you still have to wait for banking institutions to release the method of communication or a list of related hardware devices; otherwise, you are unable to conduct transactions directly with banks at present. The issues mentioned above are the main directions for future work to make the POS system become more complete, closer to the function of a traditional POS system, and more convenient to use.

\section{Conclusion}

When its hardware peripheral and functions become more efficient, and its security mechanism becomes more robust concurrently, the proposed system can completely replace a traditional POS system, which can not only greatly reduce the cost, but also bring convenience in use as the size of devices is decreased markedly. Moreover, by adding the concept of warehouse management and integrating it the result of data analysis in the future, one can learn about what products or materials are lacking at this moment in real time only by pressing a few buttons, so as to save storage space and predict future trends to help enterprises make decisions. Furthermore, the system could be linked with the supplier or logistics and one can directly place orders, which not only speeds up the purchasing and saves personnel costs, but also enhances the operating efficiency.

\section{Acknowledgments}

This work is fully supported by the Ministry of Science and Technology, Taiwan, Republic of China, under grant numbers MOST 105-2221-E-390-013-MY3 and MOST 104-2622-E-390006-CC3. 


\section{References}

1 Afuah and C. L. Tucci: Internet Business Models and Strategies, Vol. 266 (McGraw-Hill Higher Education, Columbus, 2001) p. 1. https://www.researchgate.net/publication/215915163_Internet_Business_Models_and_ Strategies_Text_and_Cases

2 P. E. Kourouthanassisa and G. M. Giaglisb: Int. J. Electronic Commerce 16 (2012) 7. https://www.researchgate. net/publication/262346446_Introduction_to_the_Special_Issue_Mobile_Commerce_The_Past_Present_and_ Future_of_Mobile_Commerce_Research

3 I. Ramos-de-Luna, F. Montoro-Ríos, and F. Liébana-Cabanillas: Inf. Syst. e-Business Manage. 14 (2016) 2. https://link.springer.com/article/10.1007/s10257-015-0284-5

4 E. P. Putra and H. Juwitasary: TELKOMNIKA 16 (2018) 2. https://search.proquest.com/openview/a5857bf218f d3b7451619b5f0108de80/1?pq-origsite $=$ gscholar\&cbl=376296

5 A. Zanella, N. Bui, A. Castellani, L. Vangelista, and M. Zorzi: IEEE Internet Things J. 1 (2014) 22. https:// ieexplore.ieee.org/document/6740844

6 L. Malina, P. Dzurenda, J. Hajny, and Z. Martinasek: Proc. 2018 41st Int. Conf. Telecommunications and Signal Processing (TSP) (IEEE, 2018) 1-5. https://ieeexplore.ieee.org/abstract/document/8441334

7 L. A. N. Jie: Comput. Knowl. Technol. 4 (2011) 59.

8 J. A. Stankovic: IEEE Internet Things J. 1 (2014) 3. https://ieeexplore.ieee.org/abstract/document/6774858

9 Android NFC Read UUID http://aayeah-android.blogspot.tw/2012/04/nfcmifare-carduid.html (accessed March 2019).

10 NFC-Enabled Cellphone Shipments to Soar Fourfold in Next Five Years: https://news.ihsmarkit.com/pressrelease/design-supply-chain/nfc-enabled-cellphone-shipments-soar-fourfold-next-five-years (accessed July 2019).

11 L. Leonardi, G. Patti, and L. L. Bello: IEEE Access 6 (2018) 26505. https://ieeexplore.ieee.org/abstract/ document $/ 8355905$

12 M. Collotta, G. Pau, T. Talty, and O. K. Tonguz: IEEE Commun. Mag. 56 (2018) 7. https://ieeexplore.iee.org/ abstract/document $/ 8419192$

13 A. Sikora, M. Krzysztoń, and M. Marks: Proc. 2018 Int. Conf. Military Communications and Information Systems (IEEE, 2018) 1-6. https://ieeexplore.ieee.org/abstract/document/8398689

14 J. G. Matusik, R. Heidl, J. R. Hollenbeck, A. Yu, H. W. Lee, and M. Howe: J. Appl. Psychol. 104 (2019) 3. http://dx.doi.org/10.1037/ap10000334

15 D. Choi and Y. Lee: IEEE Access 6 (2018) 42687. https://ieeexplore.ieee.org/abstract/document/8419696

16 E. Husni and S. Purwantoro: Proc. 2012 Int. Conf. Syst. Engineering and Technology. (IEEE, 2012) 1-6. https://ieeexplore.ieee.org/document/6339286

17 Mifare Official Website: https://www.mifare.net/ (accessed July 2019).

18 S. Challa, G. Geethakumari, and C. S. N. Prasad: Proc. 2011 Annual IEEE India Conf. (IEEE, 2011) 1-4. https://ieeexplore.ieee.org/document/6139641

19 X. Tang, C. Hu and W. X. Lin: Proc. 2015 IEEE Int. Conf. Information and Automation (IEEE, 2015) 17901794. https://ieeexplore.ieee.org/document/7279577

20 B. Charles, Management Strategies for The Cloud Revolution: How Cloud Computing Is Transforming Business and Why You Can’t Afford to Be Left Behind (McGraw-Hill, Columbus, 2010).

21 Epson Android SDK: https://download.epson-biz.com/modules/pos/index.php?page=single_soft\& cid=4747\&pcat=3\&scat=50 (accessed March 2019).

22 QR-code Wikipedia: https://en.wikipedia.org/wiki/QR_code (accessed July 2019).

23 O'Pay mobile payment: https://www.opay.tw/ (accessed July 2019).

24 Ministry of Finance, R.O.C (Taiwan): https://www.mof.gov.tw/Eng/Home (accessed July 2019).

25 W. Frisby, B. Moench, B. Recht, and T. Ristenpart: Proc. 6th USENIX Workshop on Offensive Technologies Conf. (USENIX, 2012) 22-33. https://www.usenix.org/conference/woot12/workshop-program/presentation/ Frisby 